\title{
NATURAL HISTORY OF RETINOPATHY OF PREMATURITY: A PROSPECTIVE STUDY
}

\author{
ALISTAIR R. FIELDER $*^{* 1}$, DAVID E. SHAW*1, JUDITH ROBINSON $*^{* 1}$ and YIN K. NG*2 \\ Leicester
}

\begin{abstract}
SUMMARY
The natural history of retinopathy of prematurity (ROP) has been studied prospectively in 572 infants $\leqslant 1,700 \mathrm{~g}$ birthweight using a protocol designed specifically to investigate the subtle features of this condition. Acute ROP developed in 50.9\%. All ROP stages 1 and 2 underwent complete resolution and of the $27(4.7 \%)$ infants with stage $3 / 4$ disease, cicatricial sequelae developed in six. Incidence and severity increased with decreasing birthweight and gestational age. Onset was not confined to the temporal retina but exhibited a predilection to start first in the nasal retina of the most immature neonate. The vertical retinal regions were relatively spared. Retinal arteriolar tortuosity developed around three months postnatally and was related to ROP severity but not its presence. The age at onset and rate of progression of retinopathy were largely determined by the stage of development but were also modified by systemic and local factors. The relevance of these findings to ROP screening is discussed.
\end{abstract}

The resurgence of interest in retinopathy of prematurity (ROP) over the past decade or so has resulted in an internationally agreed classification system ${ }^{1,2}$ and also the finding that cryotherapy is an effective method of treatment for severe disease. ${ }^{3,4.5}$ Yet despite this activity certain aspects of its natural history have yet precisely to be defined. The need for knowledge of the sequence of events occurring at a retinal level is twofold. First, it enables the design of appropriate and simple screening programmes. Second, it may provide further clues to the pathogenesis of this disease.

This article describes the natural history of ROP in detail using data obtained from a prospective study. This was undertaken in three cities of the East Midlands of England and the protocol was specifically designed to investigate the subtle features of acute ROP. This population was not influenced by either vitamin $E$ prophylaxis or,

From University of Leicester Medical School, Leicester* currently University of Birmingham Medical School, Birmingham ${ }^{\prime}$ and University of Nottingham Medical School, Nottingham ${ }^{2}$.

Correspondence to: Alistair R. Fielder, FRCS, FCOphth, Birmingham and Midland Eye Hospital, Church Street, Birmingham B3 2NS. once ROP had developed, by treatment with cryotherapy (except for one eye of one infant). Publications from this study include: epidemiology using a subgroup of infants born to mothers resident within defined geographical boundaries, ${ }^{6}$ a preliminary report on risk factors, ${ }^{7}$ and the age at onset and initial site of retinal involvement. ${ }^{89}$ In addition, using selected ROP retinal location data we have explored the possibility that light may be a causative factor. ${ }^{10}$

\section{PATIENTS AND METHODS}

Commencing July 1, 1985, and running until May 31, 1987, a prospective study of ROP was undertaken in the cities of Leicester, Derby and Nottingham. All infants who survived three weeks, with birthweights $\leqslant 1,700 \mathrm{~g}$ and were admitted to one of the five neonatal units of these cities were enrolled into the study. Although the majority were born to mothers locally resident, a number $(n=67)$ were transferred into this area from outlying units. No infant received supplemental vitamin $E$. One eye of one infant was treated by cryotherapy.

Ophthalmic examinations (ARF) commenced at three weeks of age and were performed weekly whilst on the neonatal unit, fortnightly following discharge from hospital, until 12 weeks of age, and thereafter as clinically indicated. All infants had a final assessment at six months corrected age. The pupils were dilated using cyclopentolate $0.5 \%$ eye drops, with or without phenylephrine $2.5 \%$ eye drops, prior to examination by indirect ophthalmoscopy with a 28 dioptre lens. Before the age of 12 weeks, following the instillation of topical anaesthesia (oxybuprocaine eye drops), an eyelid speculum and scleral indentor were also used, the latter for ocular rotation, rather than indentation. This facilitated visualisation of the peripheral retina over its entire circumference. Ophthalmic findings were recorded on a form designed specifically for this study, which included information on acute and cicatricial ROP, location by zone and clock-hour. Retinal zones were centred on the optic disc, with the radius of zone 1 extending temporally to twice the optic disc-macula distance, zone 2 involving the entire nasal retina and the corre- 
sponding area of the temporal retina, with zone 3 occupying the temporal crescent. Other signs recorded included the presence of haemorrhage, state of regression of the tunica vasculosa lentis and completion of vascularisation in the retinal temporal periphery. The degree of arteriolar tortuosity of the major vessels close to the optic disc was graded 0 to ++++ . The following signs of 'plus' disease were recorded: dilatation/tortuosity of the posterior vessels, iris vessel dilatation, pupillary rigidity and vitreous haze. Acute ROP was classified in accordance with the 1984 International Classification of Retinopathy of Prematurity ${ }^{1}$ and for cicatricial changes the classification of Reese, King and Owens of 1953 was used. " At the final visit at six months corrected age, details other than ROP, such as visual acuity (using the acuity card procedure), refractive status, the presence or absence of fusion and strabismus were all recorded, but will not be considered here.

Ophthalmic findings were entered onto the mainframe computer at Birmingham University (IBM 3090) and analysed by means of the statistical package SPSS.

The term gestational age (GA) denotes time from the last menstrual period to birth. Thereafter age is either referred to as postnatal age (PNA: age in weeks after birth) or, correcting for the degree of prematurity, postmenstrual age (PMA: gestational age+postnatal age). The term postconceptual age is also commonly used and is synonymous with PMA.

Permission for this study was obtained from the Ethical Committees of Leicestershire, Nottingham and Southern Derbyshire Health Authorities.

\section{RESULTS}

The Cohort and Ophthalmic Examinations

Five hundred and seventy-two (572) infants satisfied the

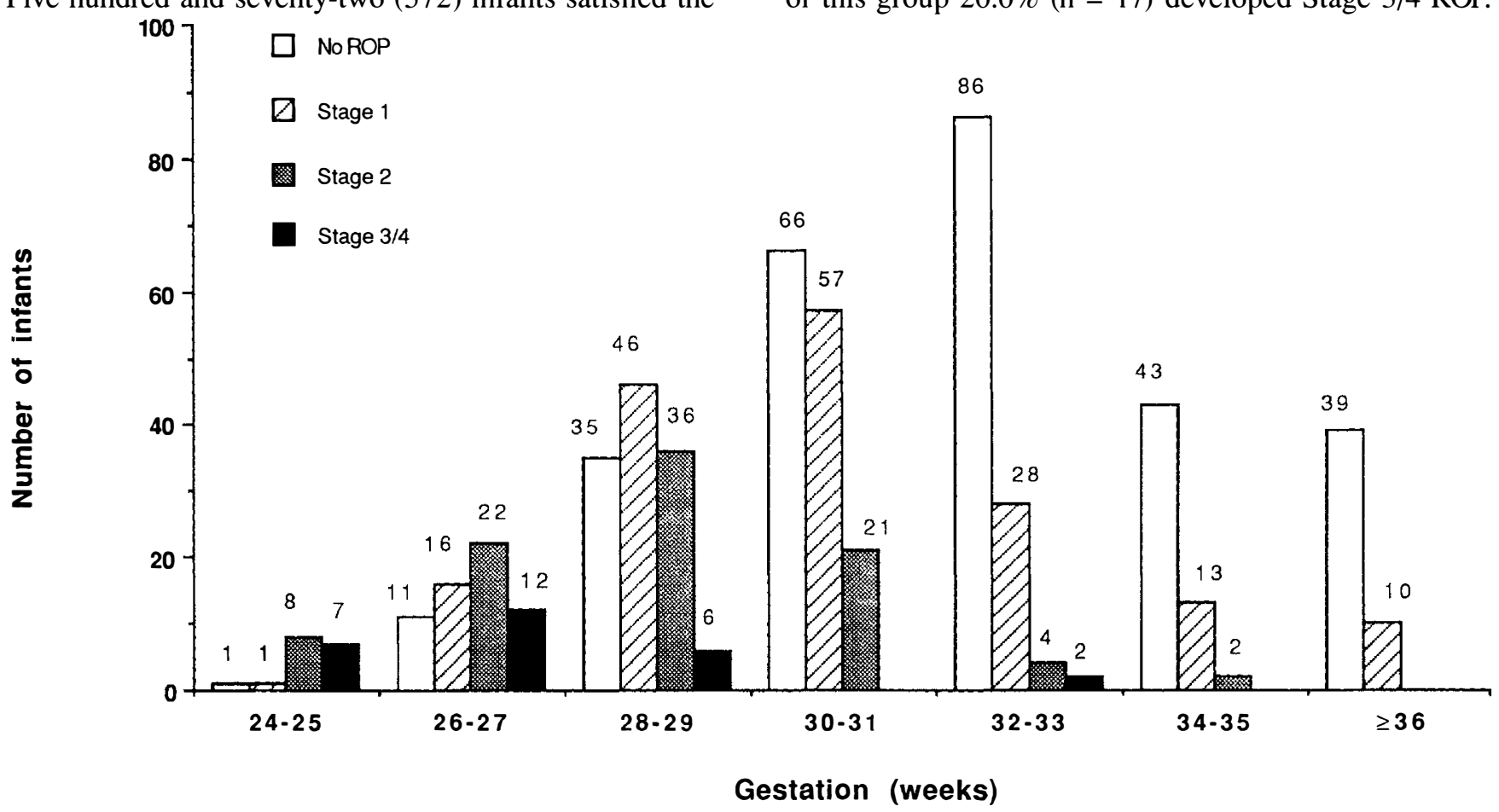

birthweight criteria and were entered into the study. Gestational age (GA) and birthweight (Bwt) distributions are shown in Figs. 1 and 2 respectively. There were 319 males and 253 females. Ethnic distribution was as follows: 465 caucasian, 66 Asian (Indo-Pakistani), 24 Afro-Caribbean and 17 others. Four hundred and fifty-one were singletons, 115 were twins and 6 the survivors of larger pregnancies.

The total number of examinations performed was 3,970 , of which $2,765(69.6 \%)$ were performed in the first 12 weeks of life. Speculum and scleral indentation were used in $80.3 \%$ of all examinations and in $98.7 \%$ before 12 weeks of age. The number of examinations per infant is shown in Fig. 3, of which 533 (93.2\%) were seen two or more times before 12 weeks. Forty-eight cases either died or moved out of the area before 12 weeks corrected age, and of the 524 available for follow-up at this age $91.8 \%$ $(n=481)$ were examined. Of the 43 who were lost to follow-up 25 had no ROP, 12 stage 1 , and 6 stage 2 at the last examination.

\section{Incidence of Acute ROP}

Acute ROP developed in 291 (50.9\%) of this cohort of 572 infants. The maximum stage reached was: 1 in 171 (29.9\%), 2 in $93(16.3 \%), 3$ in $25(4.4 \%)$, and 4 in $2(0.3 \%)$ infants. For the remainder of this article the 48 eyes of 27 infants with stages 3 and 4 will be considered together as severe disease. The distribution of acute changes according to GA and Bwt are shown in Figs. 1 and 2. Both measures of prematurity were significantly related to both the development of ROP and to the worst stage reached. Thus infants with lower Bwt or lesser GA were prone to develop severe changes. The incidence of ROP in the 85 infants of birthweight $\leqslant 1,000 \mathrm{~g}$ was $87.1 \%(\mathrm{n}=74)$ and of this group $20.0 \%(n=17)$ developed Stage $3 / 4$ ROP.

86

Fig. 1. Gestational age distribution of the cohort and maximum stage of ROP achieved. Number of infants on top of histogram bars. 


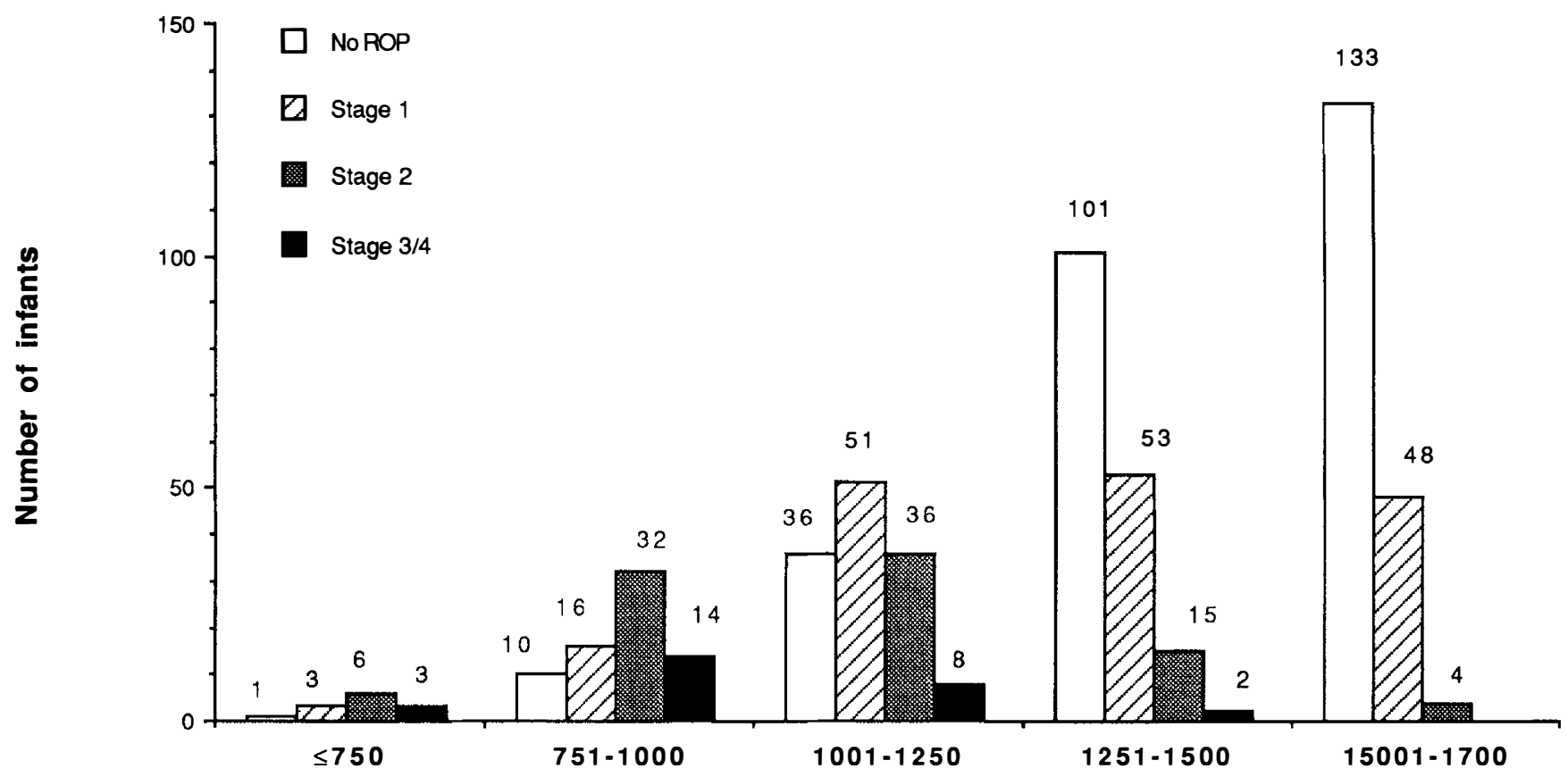

Birthweight (g)

Fig. 2. Birthweight distribution of the cohort and maximum stage of ROP. Number of infants on top of histogram bars.

For the 78 infants $\leqslant 27$ weeks GA, the corresponding figures were $84.6 \%(n=66)$ and $24.4 \%(n=19)$ with stage $3 / 4$ disease. All stage 3 cases were $\leqslant 33$ weeks GA (all but $2 \leqslant 29$ weeks), and $\leqslant 1,425 \mathrm{~g} \mathrm{Bwt}(17 \leqslant 1,000 \mathrm{~g}$ and all but $2 \leqslant 1,250 \mathrm{~g}$ ). Two infants developed stage 3 , one each of 32 and 33 weeks GA, but with Bwts of $525 \mathrm{~g}$ and $1,200 \mathrm{~g}$ respectively. Incidence and severity were similar for each sex: of 319 males, 150 had no ROP, 95 stage 1,57 stage 2 and 17 stage 3 . For the 253 females the corresponding figures were $131,76,36$ and 10 . There was no significant difference in ROP incidence or stage between the sexes $(\mathrm{p}>0.05)$.

ROP stage according to ethnic origin is tabulated in Table I. Despite a similar overall incidence, there was a significant disproportion of Asian children developing the most severe disease. ${ }^{6}$ Although numbers are small, Afro-

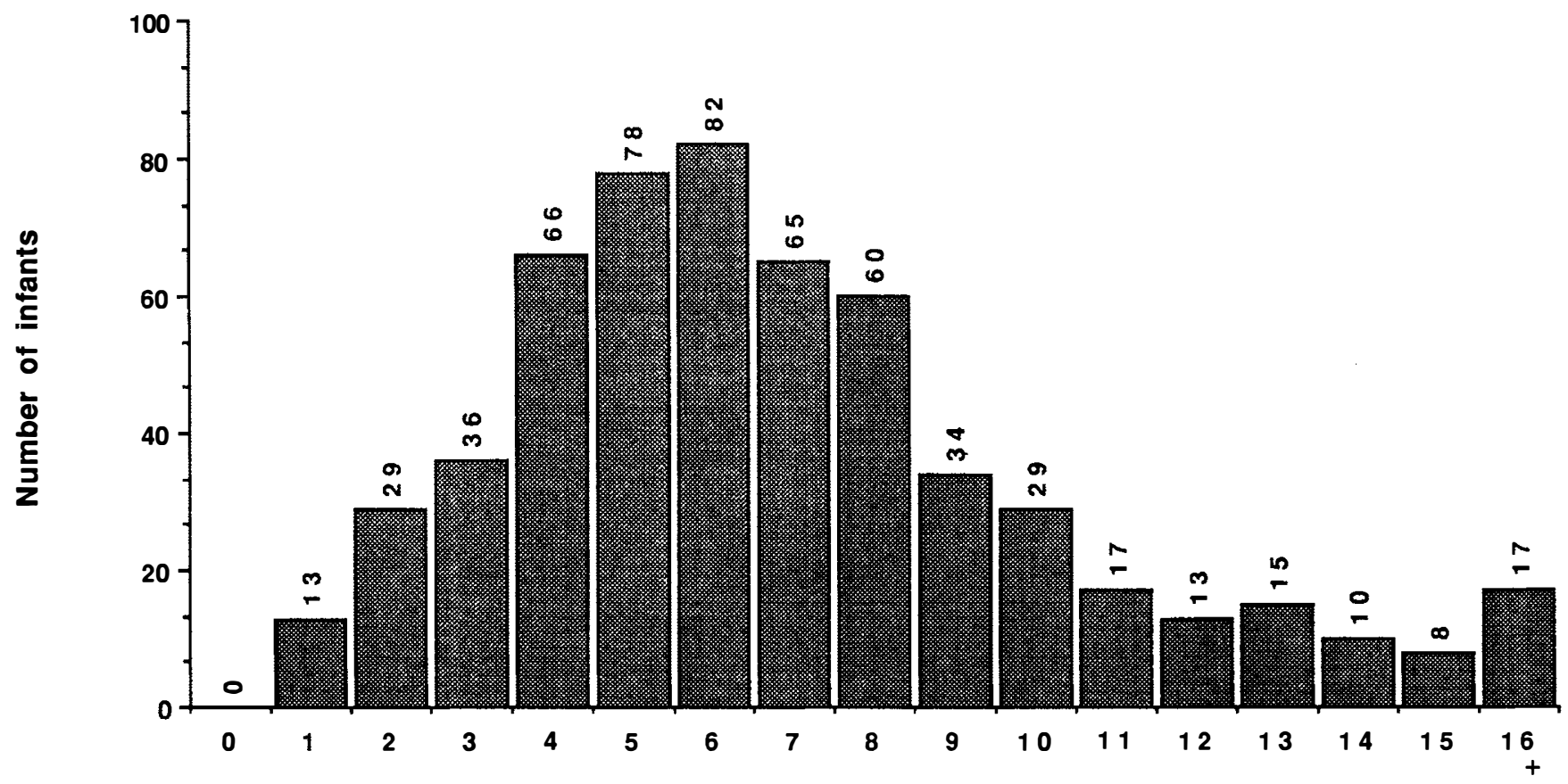

Total number of examinations

Fig. 3. Total number of examinations. Numbers on top of bars indicate number of infants who received that number of examinations. 
Table I Ethnic origin and ROP stage. See text for details

\begin{tabular}{lrrrr}
\hline & \multicolumn{4}{c}{ ROP stage } \\
\cline { 2 - 5 } Ethnic origin & \multicolumn{1}{c}{0} & \multicolumn{1}{c}{1} & 2 & $3 / 4$ \\
\hline Caucasian & 223 & 148 & 77 & 17 \\
Asian & 33 & 13 & 11 & 9 \\
Afro-Caribbean & 15 & 6 & 2 & 1 \\
Other & 10 & 4 & 3 & 0 \\
\hline
\end{tabular}

Caribbean infants were the least likely to develop any ROP.

Multiple pregnancies accounted for 157 infants including one set of triplets and three survivors of quadruplets. This group contained 35 siblings with $\mathrm{Bwt}>1,700 \mathrm{~g}$ who were in the study but have not been included, with this exception, in the analysis. There were 17 sole survivors. Thirty-two sets of twins and one set of triplets had no ROP $(n=67)$. Eight sets of twins developed retinopathy with each twin behaving identically with regard to age at onset and maximum ROP stage. In the remaining 27 sets of twins and the three surviving quadruplets $(n=57)$, within each set each infant did not behave similarly. Of these, there were 17 sets in which only one member developed ROP and in 14 sets the infant of lower birthweight was affected.

\section{Site of Onset}

There were regional variations of ROP at onset and the

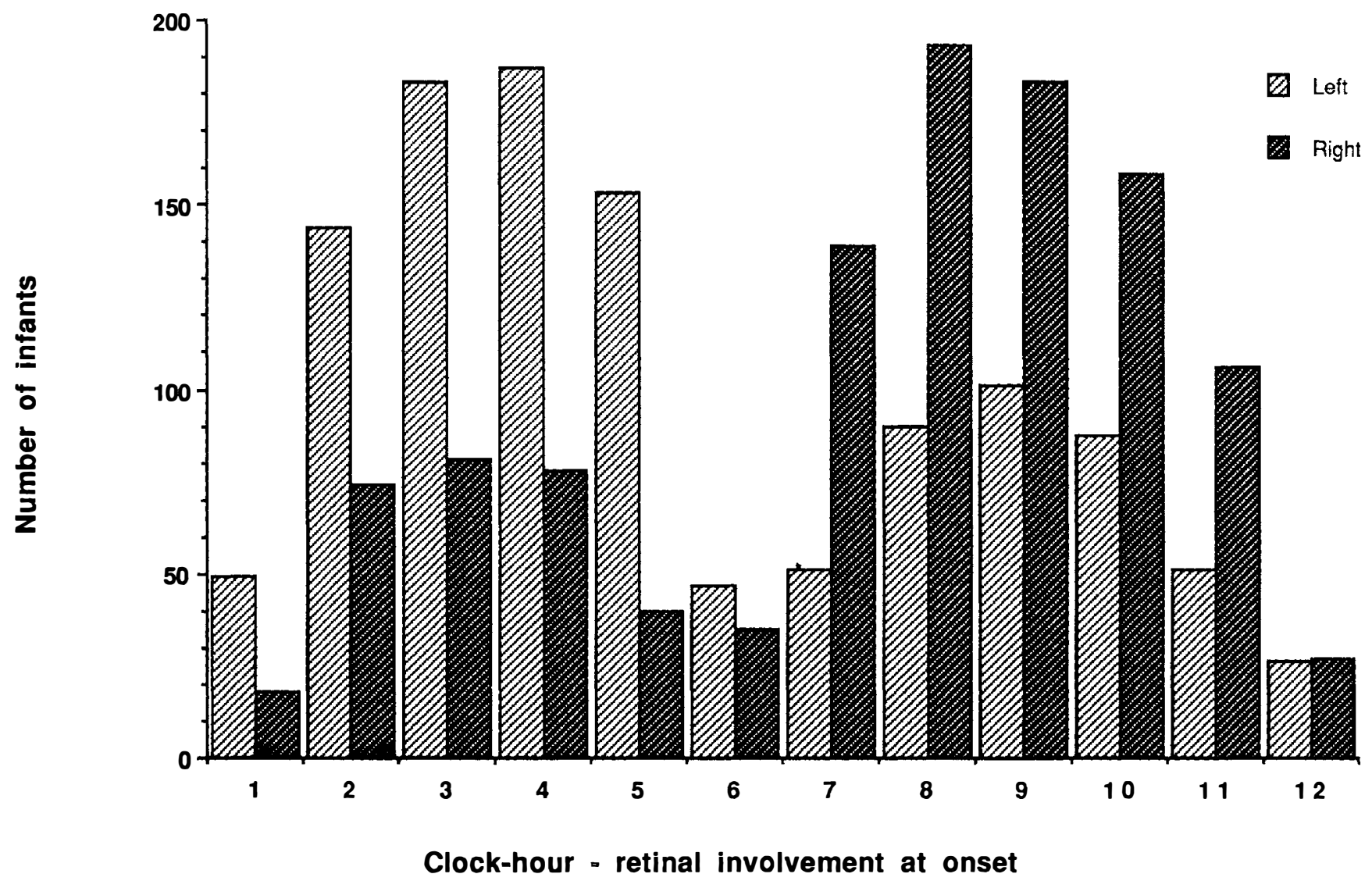

process exhibited a propensity to involve the nasal and temporal retina rather than the superior and inferior regions (Fig. 4). The horizontal retinal regions where acute changes were first visualised, subdivided according to GA and Bwt are shown in Figs. 5 and 6. Site of onset was significantly related to the degree of prematurity (median GA), as follows: no ROP 32.0 weeks; temporal onset ROP, 30.0 weeks; onset in the temporal and nasal retina simultaneously, 28.0 weeks; inferior, 29.0 weeks; in the nasal retina alone 28.0 weeks, and superior retina 27.5 weeks GA $(\mathrm{p}<0.0001)$. Thus the relatively mature infant developed ROP initially in the temporal retina, but with decreasing GA there was a tendency for the retinopathy to develop either in both the nasal and temporal retina simultaneously, or in the most immature infant solely in the nasal retina.

Most $(n=25)$ of the 27 cases which later progressed to stage 3 disease exhibited changes initially either solely in the nasal retina $(\mathrm{n}=11)$ or simultaneously in the nasal and temporal retina $(n=14)$, with only two starting temporally (both having subsequent nasal involvement). Of those with initial changes in the nasal retina $(n=54)$, 94.4\% $(n=51)$ developed temporal ROP later, whilst of those with temporal onset $(n=171), 18.7 \%(n=32)$ had subsequent nasal development. ROP was less likely to involve the vertical regions, and in no infant did ROP at onset involve solely 6 or 12 o'clock.

\section{Age at Onset}

The postnatal age at which acute retinopathy developed 


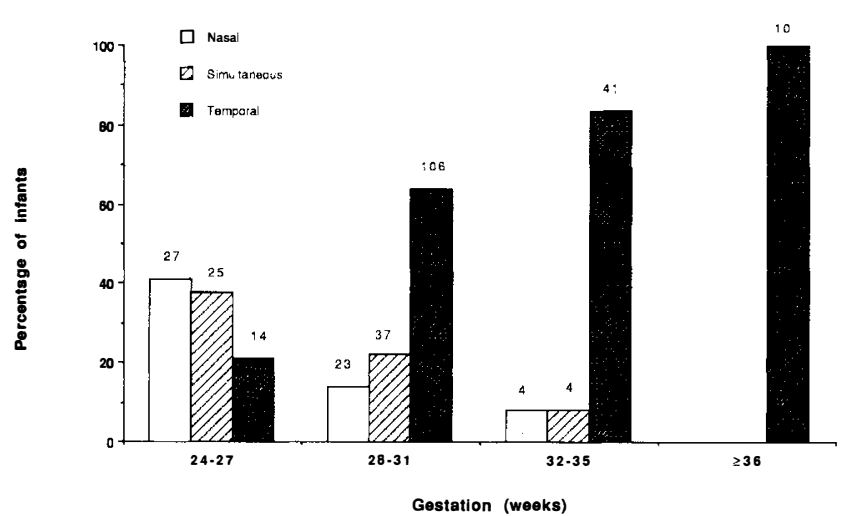

Fig. 5. Site of onset of ROP along the horizontal meridia according to $G A$. In the relatively mature infants retinopathy has a predilection to commence in the temporal retina, but with increasing immaturity there is tendency for this to be observed first in either the nasal-temporal regions simultaneously or in the most immature in the nasal retina alone.

was inversely proportional to both decreasing Bwt ( $p=0.028$, Kruskal Wallis) and GA ( $p=0.0003$ Kruskal Wallis). The single exception being the 1,500-1,700 $\mathrm{g}$ group with a median age at onset of 42 days, greater than all but the $\leqslant 750 \mathrm{~g}$ group. This probably reflects the difficulty of following closely the larger infant discharged from hospital relatively soon after birth.

The PMA at which ROP developed ranged from 29.7 to 45.0 weeks (Fig. 7): $92.1 \%$ between 30.0 and 40.0 weeks; $80.8 \%$ between 30.0 and 37.8 weeks; $70 \%$ between 30.0 and 36.0 weeks; and $65 \%$ between 30.0 and 35.0 weeks PMA. ROP developed after 40 weeks PMA in 23 infants; GAs 31 to 38.0 weeks, except for one of 27 weeks GA in whom retinopathy was first diagnosed at 41.6 weeks PMA. In this case there had been no examination in the previous five weeks. Of these 23, 21 had stage 1 , and 2 stage 2 ROP.

The degree of prematurity conferred a degree of hastening of ROP onset $(\mathrm{R}=0.632)$. The median PMA at onset by retinal location for left and right eyes was as follows: temporal 35 weeks (interquartile range, IQR, 34-37); inferior 35, nasal 33 and superior 34 weeks PMA.

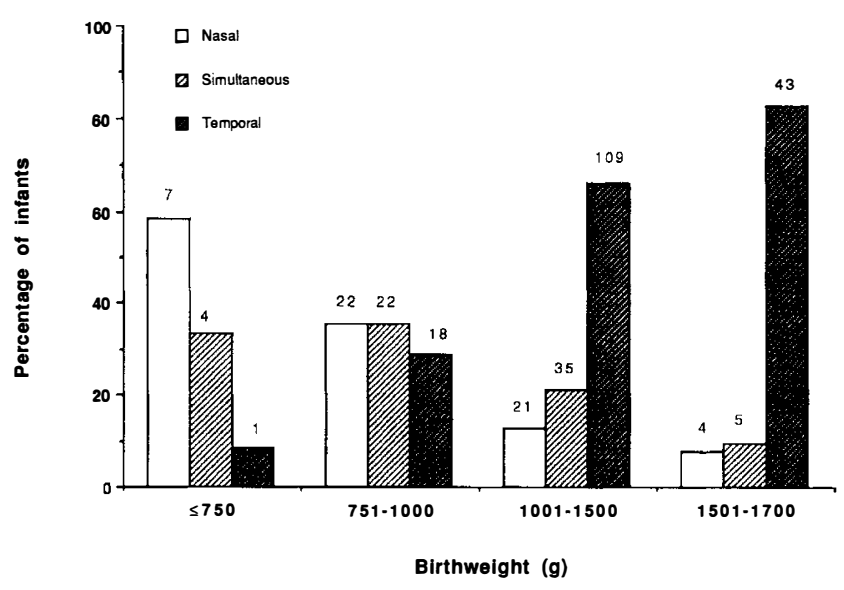

Fig. 6. Site of onset of ROP along the horizontal meridia according to Bwt. See Fig. 5 for explanation.

\section{Clock-hour involvement}

As shown in Fig. 8 the propensity of ROP to extend circumferentially varied according to site of involvement at onset and increased as follows: temporal $<$ inferior $<$ nasal<superior. Of the 48 eyes of 27 infants with Stage 3, 27 had $\geqslant 5$ clock hour involvement.

\section{Zone of Involvement}

Zone I involvement was seen in four right (11 left eyes) and zone II in 130 right eyes (120 left), and 125 right eyes (135 left) had zone III involvement alone. Of the 48 eyes that progressed to stage 3 disease, five had zone I involvement and the remainder (43) had zone II involvement. No stage 3 cases had disease confined to zone III. Twentyseven (27) cases demonstrated asymmetry of zone at the first signs of ROP.

The median Bwts and GAs by zone were as follows zone 1: $940 \mathrm{~g}(843-1,024 \mathrm{~g})$ and 26 weeks GA (25-28 weeks), zone 2: 1,050 g (900-1,243 g) and 28 weeks GA (27-30 weeks), and zone 3: 1,360 g (1,158-1,540 g) and 30 weeks GA (29-32 weeks).

\section{ROP Symmetry}

In 74 of the 291 cases acute ROP was asymmetrical, and in all by only one stage. Using $\mathrm{N}(\mathrm{R}, \mathrm{L})$ notation, where $\mathrm{N}=$ number of cases exhibiting ( $\mathrm{R}$ stage, $\mathrm{L}$ stage), the asymmetry was: $28(0,1), 21(1,0), 10(2,1), 9(1,2), 2(3,2)$ and $4(2,3)$, and the symmetry: $281(0,0), 122(1,1), 74(2,2)$ and 21(3,3). all six cases of cicatricial ROP exhibited asymmetry which in four exceeded 1 stage: $1(2,3), 1(3,2)$, $1(3,0), 2(3,1)$ and $1(2,0)$.

\section{Progression and Resolution of Acute ROP}

Figure 7 shows the PMA at which the various stages of acute ROP developed. Stage 1 disease developed at a median PMA of 34.6 weeks (IQR 32.7-37.0 weeks); stage 2, 35.1 weeks (IQR 33.1-36.8 weeks); and Stage 3, 37.1 weeks (IQR 35.6-39.0 weeks). Stage 3 was first seen in two infants $\geqslant 42$ weeks PMA. In one infant this was in the 42nd week, the previous examination having been two weeks before, while in the other, Stage 3 was diagnosed at 47 weeks PMA, with the previous examination four weeks before.

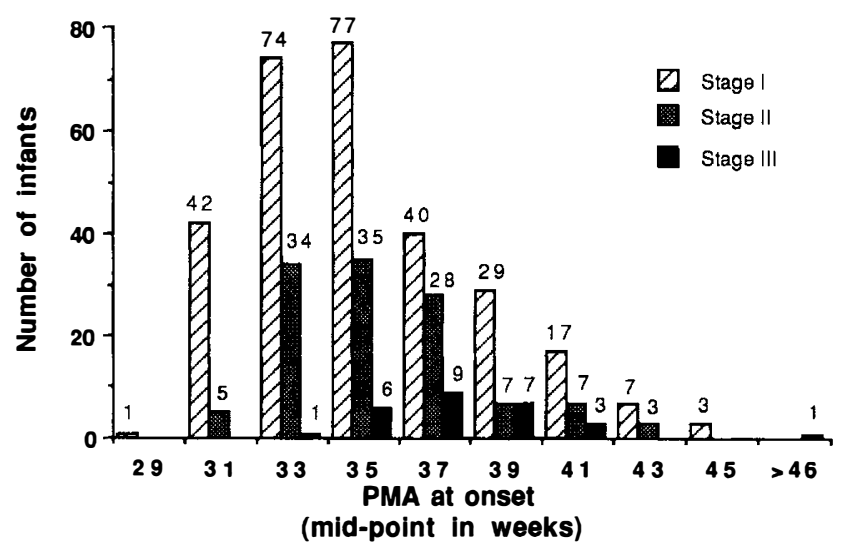

Fig. 7. Onset of acute ROP and progression according to postmenstrual age (PMA). Presented as mid-point in weeks. 


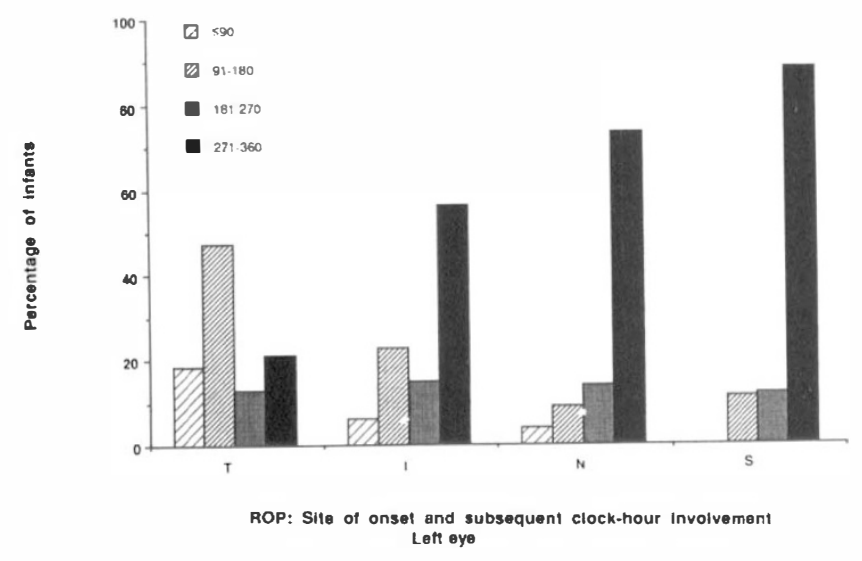

Fig. 8. Site of ROP onset and subsequent clock-hour extension. The likelihood of circumferential extension increases according to the retinal site of onset: temporal $<$ inferior $<$ nasal $<$ superior $($ TINS).

The PMA at which the various stages of acute ROP underwent regression is shown in Fig. 9. The median PMA at which the acute phase resolved was 39.7 weeks (IQR 37.0-43.9 weeks). All stage 1 and 2 changes underwent complete resolution. Stage 3/4 disease involved 48 eyes of 27 infants and was unilateral in six. Cicatricial disease therefore resulted only from stage $3 / 4$ ROP and developed in 10 eyes of six infants $(880-1300 \mathrm{~g} \mathrm{Bwt}$; 25-28 weeks GA): grade I in six eyes, grade II in 1, and grade III in three eyes. Only one eye of one infant was treated with cryotherapy and this eye subsequently developed stage 3 cicatricial disease.

\section{'Plus Disease}

Dilatation/congestion of the posterior vessels was observed in 27 infants, all of whom also had acute ROP, the worst stage being stage 1 in two, stage 2 in six and stage 3 in 19. For stage 3 this was the most frequent indicator of 'plus' disease; the only one in eight cases, and in no case were other signs of 'plus' present without this indicator. Iris vessel engorgement was seen in 14 cases, stage 3,10 cases; stage 2 , one, and stage 1 , one case; no ROP, two cases. Pupil rigidity developed in 10 cases of stage 3. Vitreous haze was noted in 79 infants in the first few weeks after birth, but only associated with stage 3 in one baby. Forty-eight (48) infants had retinal haemorrhages; 24 pre-ROP onset, 15 of which subsequently developed stage 3 disease.

\section{White Patches}

In 79 infants white patches were observed in the region of the tips of the retinal vessels in the superficial retina on one or more of the examinations. Sometimes these patches were small and isolated, on other occasions they were confluently arranged circumferentially, and with regression were traversed by the peripherally growing vessels. Twenty-four (24) of these had no ROP, 24 stage 1, 20 stage 2 , and 11 stage 3 . The median postnatal ages at which they were observed, in days, by presence and stage of ROP were: 0,$57 ; 1,70 ; 2,105 ; 3,104$ days.

\section{Arteriolar Tortuosity}

Retinal arteriolar tortuosity (AT) was not observed early on and the minimum postnatal age (in days) at which this appeared was for the right eye, (left eye): AT+111(110); AT++170 (171); AT+++143 (153); AT++++81 (81). At three months corrected age $46(8 \%)$ right eyes had no arte-

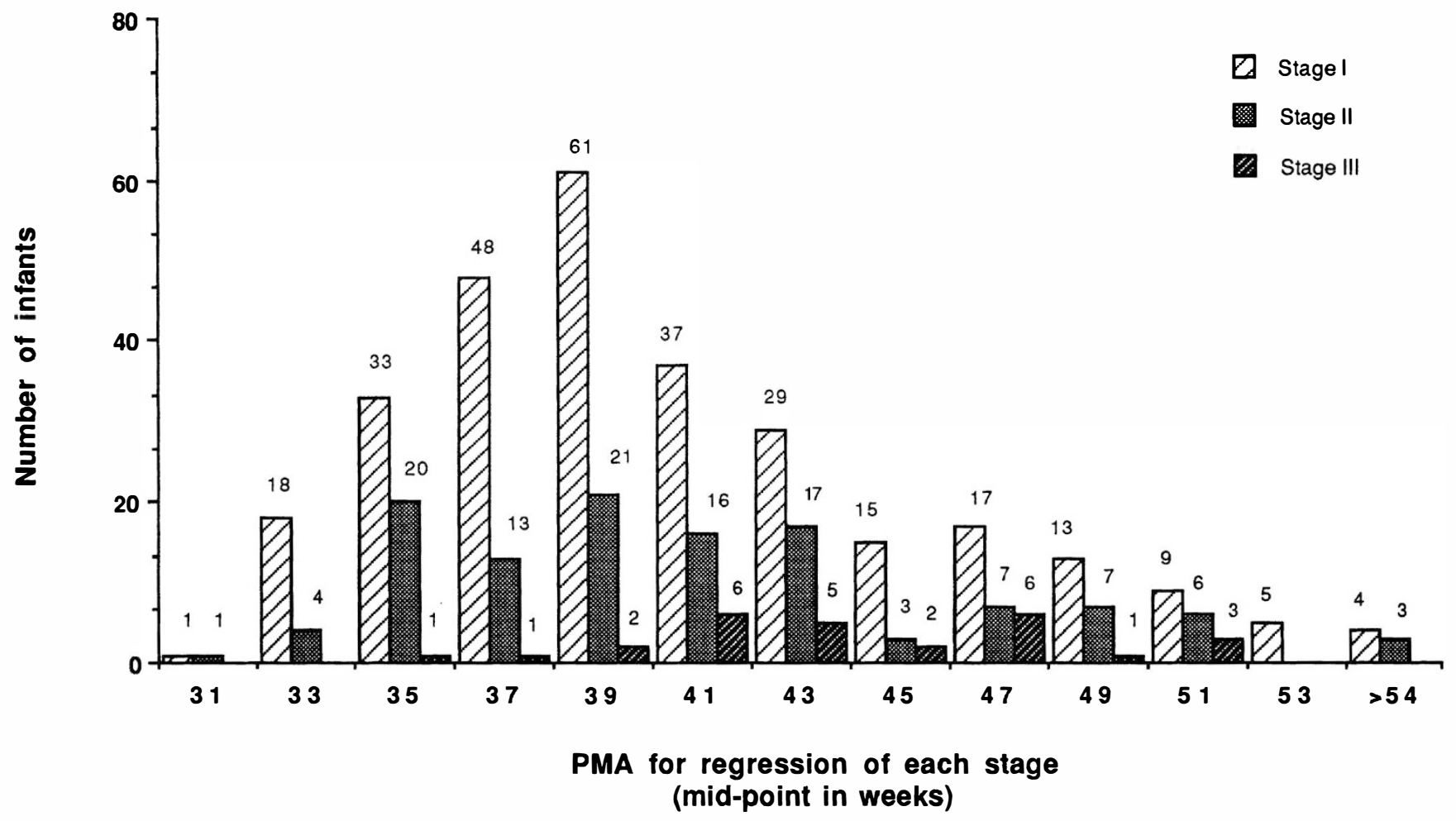

Fig. 9. Postmenstrual age for regression of each stage of acute ROP, presented as mid-point in weeks. 
riolar tortuosity, 193 had,$+ 274++, 54+++$, and $5++++$. The degree of arterial tortuosity was significantly related to the worst stage of ROP thus for the 46 right eyes exhibiting no AT, $11(23.9 \%)$ had ROP and none had stage 3 . Of the 193 with AT+58 $(30.1 \%)$ had ROP with none at Stage 3: of the 274 with AT++151 (55.1\%) had ROP and $9(3.3 \%)$ stage 3 disease, and 54 with AT +++37 $(68.5 \%)$ had ROP and $9(16.7 \%)$ stage 3 . All five with AT++++ were stage 3 cases, coinciding with the onset of 'plus' disease.

The degree of tortuosity at a corrected age of six months was available for 469 infants. Four cases had no tortuosity, 196 had AT+, $246 \mathrm{AT}++$ and 23 had AT+++, and none AT++++.

\section{DISCUSSION}

The natural history of ROP has been studied prospectively in 572 infants of Bwt $\leqslant 1,700 \mathrm{~g}$. Before discussing issues arising from the results, certain strengths and limitations of this study will be identified. The study protocal was specifically designed to investigate the subtle features of acute ROP. Examinations commenced relatively early postnatally, were performed regularly until the likelihood of ROP development had passed (completion of retinal vascularisation), and involved scrutiny of the entire retinal circumference. This permitted the identification of certain of the more transient signs of ROP such as its onset, clockhour location, and progression rate, which would not have been observed in a less rigorous programme. Being undertaken in a geographically defined area the cohort also provided the basis for one of only two recent epidemiological studies. ${ }^{6,12}$ However, despite the relatively large cohort the numbers in certain categories, particularly for severe disease, were too small for statistical analysis.

It is established that the incidence and severity of retinopathy both rise with decreasing birthweight and gestational age. As our present cohort $(n=572)$ contained only a few infants in addition to the epidemiology cohort ${ }^{6}$ $(n=505)$ it is not surprising that the two sets of incidence figures do not differ significantly. Our incidence figures, particularly for the acute ROP stages are consistently higher, whilst those for cicatricial disease are lower, compared to other studies. ${ }^{13-18}$ Possible explanations have been previously debated, ${ }^{6,19,20}$ but almost certainly reflect the rigorous examination protocol used rather than issues of interpretation of ophthalmoscopic findings, or true variation of incidence. Similarly differences in the incidence and severity across ethnic groups have been discussed..$^{6,21,22}$

Scant attention has been paid to the site of ROP onset. Traditionally retinopathy was assumed to commence always in the temporal retina, ${ }^{23-28}$ this being the last region to vascularise. In previous decades this may well have been so, as the neonate at risk of developing severe ROP (ie at risk of blindness) was in the 1,000-1,500 $\mathrm{g}$ birthweight group, whereas nowadays it is mainly confined to those of birthweight $750-999 \mathrm{~g},{ }^{29.30}$ who in the past were unlikely to survive. Nevertheless there are anecdotal refer- ences to acute changes in the nasal retina ${ }^{25,28}$ and early on in this study ${ }^{8}$ we were surprised to find that not only did ROP not always commence temporally, but it had a predilection in the most immature to start first in the nasal retina. Here these findings are confirmed. Nissenkorn et al. recently ${ }^{31}$ reported stage 3 disease developing two weeks earlier in the nasal compared to the temporal retina. However the results of this study ${ }^{10}$ show (Fig. 4) for the first time that the regional variations in the onset of ROP are not confined to the horizontal meridia. Thus ROP tends to spare the superior and inferior retinal areas, and when they are involved its onset at these sites is frequently delayed. ${ }^{10}$

The location of ROP by zone (antero-posterior location) and clock-hour (circumferential location) is predictive of future progression. With respect to zone, the more posterior the process, the greater the potential for severe disease. Hence the poor prognosis for zone 1 disease ${ }^{4}$ not surprising as the zone of involvement is an indication of the degree of retinovascular immaturity and consequently its potential for progression. In this study no ROP confined to zone 3 achieved stage 3 disease. Clockhour involvement at onset indicated the likelihood of subsequent extension around the circumference (Fig. 8). Thus about $20 \%$ of ROP confined to the temporal retina at onset, later extended to involve the entire circumference, whereas for the nasal and superior retina this rose to about 75-90\%. Acute ROP tended to be symmetrical and the two eyes did not differ by more than one stage in this study. However clock-hour asymmetry occurred more frequently, perhaps accounting for the characteristic asymmetry of cicatricial disease. ${ }^{32}$

This study also confirms previous observations ${ }^{18,33,34}$ that ROP onset occurs at a later postnatal age in more immature neonates compared with their relatively more mature counterparts. When correction is made for the degree of prematurity ROP develops over a relatively narrow postmenstrual age range. However as intimated by Fielder et $a l .{ }^{33}$ and highlighted by Quinn et al., ${ }^{34}$ its onset is slightly accelerated in the most immature, indicating that although the stage of development at birth is the major determining factor, the event of birth and/or postnatal events also exert some influence. Unfortunately our data only indicate a trend and greater numbers are required fully to unravel this aspect. Thus both age at onset of ROP and its rate of progression appear to be largely governed by the stage of development (Fig. 6), but this cannot fully explain all aspects as extreme prematurity probably exerts a slight hastening effect. Furthermore, not all neonates with the same degree of immaturity develop retinopathy, and those that do, do not all exhibit changes of similar severity. Other factors must be involved therefore, to account for these aspects and for the regional variations with regard to both retinal location and time of onset. The many systemic ROP risk factors including supplemental oxygen have not been considered here, but it is interesting to note that vitamin $\mathrm{E}$ prophylaxis has recently been reported to delay both ROP onset and progression. ${ }^{36}$ In view of the regional variations reported here, local events must be 
relevant. Mechanisms are unknown but the possibilities include: regional variations of retinal vascular and neural development. ${ }^{10}$ The possibility of environmental influences of ROP production, such as regional variations of the retinal light dose has recently been discussed. ${ }^{10}$ To summarise, the onset and rate of progression of the ROP process (a response of the immature retinovascular system) is largely determined by the stage of development, but may also be modified by systemic and local factors. While ROP severity is also obviously related to the degree of immaturity, systemic factors ${ }^{33}$ assume far greater importance and local factors should also be considered. ${ }^{10}$

'Plus' disease (iris vascular engorgement, pupil rigidity, posterior venous engorgement and arteriolar tortuosity) is an indicator of a particularly florid ROP response, or in the words of the Committee for the Classification of ROP 'progressive vascular incompetence'.' In this study changes in the posteriior pole vessels were the most commonly observed 'plus' sign and in no case were other activity indicators present without this sign. Although isolated signs were observed without stage 3 ROP, the constellation of 'plus' signs only occurred in eyes at this stage, or in eyes which later developed stage 3 . Thus 'plus' disease developed either before or at stage 3. Again our numbers were too small to determine the precise relation of 'plus' signs and ROP severity. Although most cases of stage $3(19 / 27)$ had at least one 'plus' sign with a trend to more signs with increasing clock-hour involvement, this was not an invariable association.

To the best of our knowledge this is the first study to report retinal white patches in preterm neonates. These are small and superficially located, just peripheral to the growing tips of the retinal vessels. They were seen in neonates with and without ROP, although in the latter the possibility of a subtle demarcation line not being identified cannot be excluded-i.e. they represent part of the regression process. Nevertheless the age at which they were observed, and that the peripherally growing vessels passed through them, suggests that they are part of the resolution process for those with ROP, and associated with the growth of vessels across the peripheral retina in those without retinopathy.

In the early neonatal period retinal arterioles do not exhibit any tortuosity, this sign develops around three months postnatally. The degree of tortuosity, but not its presence, was related to severity of acute ROP, as this sign was present in $92 \%$ whilst retinopathy developed in only $50 \%$ of our cohort. By six months corrected age arteriolar tortuosity was present in all but four infants. These findings disagree with those of Baum who suggested ${ }^{36}$ that this represents a persistent sign of the 'proliferative phase of RLF'.

As, at last, there is an effective mode of treatment for severe ROP ${ }^{3-5}$ screening has become a priority and has been addressed on a number of occasions. ${ }^{37-45}$ This study indicates that appropriate design of an ROP screening protocol is dependent on an understanding of its natural history. Guidelines are a safety net and have to attempt to foresee the unforeseen, without increasing the workload disproportionately. It is important also that guidelines do not rely too heavily on one study, which may not truly reflect other populations. With these caveats a few comments will be made from our study on two aspects. First, the selection of babies. Severe ROP affects the most immature neonates, all but two in our study were $<29$ weeks GA and all but two $\leqslant 1,250 \mathrm{~g}$ Bwt. In our opinion, due to the occasional discrepancy between birthweight and gestational age, a screening procedure should include both parameters of prematurity. For example, both infants in this study with stage 3 who were $>29$ weeks GA (32 and 33 weeks GA) had low birthweight (525 and 1,200 g) and would have been included had the birthweight criteria been set at 1,500 g. Indeed it could be argued that a birthweight criteria alone is required as this is the more powerful predictor of ROP. Our multiple birth data support this suggestion as in those with only one member affected, in most $(14 / 17)$ the one with ROP had the lowest Bwt. Gestational age, however, encourages the clinician to think in terms of the temporal aspects of the developmental sequence, and for the time being it is probably safer to include this parameter in a screening protocol. Second, the timing of ophthalmic examination. Here the fundamental principle is that the onset and progression of ROP are both governed predominantly by the stage of development rather than postnatal events. As shown in Fig. 7 the sequence of events correlates better with age corrected for the degree of prematurity (PMA) rather than postnatal age (PNA). This simplifies screening programme design considerably. Whilst recognising the importance of neonatal events as factors which influence ROP severity; as they are not major determinant factors in its timing; they do not have to be accounted for in the timing of examinations-or indeed in any part of the selection process. Timing is critical for two reasons. First, if undertaken very early on examination may precede ROP onset. Second, the time window available for cryotherapy for threshold disease ( $\geqslant 5$ hours contiguous or $\geqslant 8$ hours cumulative stage 3 ROP with 'plus' disease) is narrow, only about two to three weeks. Therefore an initial examination performed following discharge and after term may be too late. However, important clues can be gained from the normal examination as to whether further evaluation is necessary, i.e. answering the question: has the eye the propensity to develop severe disease? In this respect assessing the width of the peripheral avascular zone is useful-is the vascular/ avascular junction in zones 1,2 , or 3? Determining the first is straightforward, but in the absence of a landmark which permit zones 2 and 3 to be distinguished in the temporal retina, this can only be known with certainty by the state of vascularisation of the nasal retina. If the nasal retina is fully vascularised zone 3 has been entered, and in this study no stage 3 disease was confined solely to this zone. If the nasal retina has yet to be vascularised, severe disease can still develop. In this study no ROP which developed after term subsequently became severe. When ROP has developed, a sign which indicates a likelihood 
for progression is involvement of other areas than the temporal retina (see Fig. 7). Due to the difficulty of scrutinising the extreme retinal periphery, we consider the use of an eyelid speculum and scleral indenter important aids to examination. In the UK guidelines have recently been drawn up..$^{45}$ These recommend inclusion criteria of Bwt $<1,500 \mathrm{~g}$ and $\leqslant 31$ weeks GA, and that examinations should commence at 6-7 weeks postnatally and be undertaken fortnightly until 36 weeks PMA, or until vascularisation is into zone 3. In most infants one examination should suffice. $^{45}$

The findings of this study emphasise that low birthweight identifies the babies at risk, those who need to be screened. Postmenstrual age on the other hand largely determines when ROP will develop and progress, and thus indicates when examinations should commence, and the age at which treatment is likely.

In this study the natural history of ROP has been investigated. The intensive protocol permitted the kinetics of this condition to be studied, and due to the advent of cryotherapy, such investigations will not be possible again in the foreseeable future. We have shown that ROP onset and progression are linked to the stage of development of the infant and that retinopathy exhibits characteristic regional variations. The relevance of these findings to the routine ROP screening has been discussed. Their value as clues to our understanding of fundamental mechanisms awaits further study.

We thank paediatric and nursing colleagues at the neonatal units in Leicester, Derby and Nottingham for their help and encouragement. YKN was supported by the Medical Research Council and JR by the Royal National Institute for the Blind.

Key words: Retinopathy of prematurity, human, infant preterm, natural history, prospective study, retinal arteriolar tortuosity.

\section{REFERENCES}

1. Committee for the Classification of Retinopathy of Prematurity: An international classification of retinopathy of prematurity. Arch Ophthalmol 1984, 102: 1130-4.

2. Committee for the Classification of Retinopathy of Prematurity: An international classification of retinopathy of prematurity. II The classification of retinal detachment. Arch Ophthalmol 1987, 105: 906-12.

3. Cryotherapy for Retinopathy of Prematurity Cooperative Group: Multicenter trial of cryotherapy for retinopathy of prematurity: preliminary results. Arch Ophthalmol 1988, 106: 471-9.

4. Cryotherapy for Retinopathy of Prematurity Cooperative Group: Multicenter trial of cryotherapy for retinopathy of prematurity: three-month outcome Arch Ophthalmol 1990, 108: 195-204.

5. Cryotherapy for Retinopathy of Prematurity Cooperative Group: Multicenter trial of cryotherapy for retinopathy of prematurity: one year outcome. Arch Ophthalmol 1990, 108: 1408-16.

6. Ng YK, Fielder AR, Shaw DE, Levene MI: Epidemiology of retinopathy of prematurity. Lancet 1988, ii: 1235-8.

7. Ng YK, Fielder AR, Shaw DE, Levene MI: Acute retinopathy of prematurity: risk factors. Arch Dis Child 1988, 63: $693-4$.

8. Fielder AR, Ng YK, Levene MI, Shaw DE: Retinopathy of prematurity: age at onset and the initial site of involvement.
A preliminary report. In BenEzra D, Ryan SJ, Glaser BM, Murphy RP eds Ocular circulation and neovascularisation. Martinus Nijhoff/Dr W Junk Publishers 1987, 50: 147-54.

9. Fielder AR, Ng YK, Shaw DE, Levene MI: Retinopathy of prematurity: natural history. Invest Ophthalmol Vis Sci 1988, 29: (supplement) 121.

10. Fielder AR, Robinson J, Shaw DE, Ng YK, Moseley MJ: Light and retinopathy: does retinal location offer a clue? Pediatrics (In press).

11. Reese AB, King MJ, Owens WC: A classification of retrolental fibroplasia. Am J Ophthalmol 1953, 36: 1333-5.

12. Darlow BA: Incidence of retinopathy of prematurity in New Zealand. Arch Dis Child 1988, 63: 1083-6.

13. Keith CG and Kitchen WH: Retinopathy of prematurity in extremely low birthweight infants. Med J Aust 1984, 141: 225-7.

14. Reisner SH, Amir J, Shohat M, Krikler R, Nissenkorn I, Ben-Sira I: Retinopathy of prematurity: incidence and treatment. Arch Dis Child 1985, 60: 698-701.

15. Schaffer DB, Johnson L, Quinn GE, Weston M, Bowen FW: Vitamin E and retinopathy of prematurity. Ophthalmology 1985, 92: 1005-11.

16. Flynn JT, Bancalari E, Bachynski BN, Buckley EB, Bawol $\mathrm{R}$, Goldberg R, Cassidy J et al:: Retinopathy of prematurity. Diagnosis, severity and natural history. Ophthalmology 1987, 94: 620-9.

17. Schulenberg WE, Prendiville A, Ohri R: Natural history of retinopathy of prematurity. $\mathrm{Br} J$ Ophthalmol 1987, 71: 837-43.

18. Fledelius HC: Retinopathy of prematurity. Clinical findings in a Danish County 1982-1987. Acta Ophthalmol 1990, 68: 209-13.

19. Darlow BA: Retinopathy of prematurity (letter). Lancet 1989, i: 275.

20. Fielder AR, Shaw DE, Ng YK: Retinopathy of prematurity (letter). Lancet 1989, i: 275-6.

21. Monos T, Yassur Y, Lifchitz T, Shani L, Zmora E, Karplus M: ROP in Soroka medical center neonatal ICU. In BenEzra D, Ryan SJ, Glaser BM, Murphy RP eds Ocular circulation and neovascularisation. Martinus Nijhoff/Dr W Junk Publishers 1987, 50: 193-6.

22. Charles JB, Ganthier R, Appiah AP: Incidence and characteristics of retinopathy of prematurity in a low-income inner-city population. Ophthalmology 1991, 98: 14-17.

23. Kinsey VE: Retrolental fibroplasia: cooperative study of retrolental fibroplasia and the use of oxygen. Arch Ophthalmol 1979, 56: 481-529.

24. Flynn JT, O'Grady GE, Herrera J, Kushner BJ, Cantolino S, Milam W: Retrolental fibroplasia. 1. Clinical observations. Arch Ophthalmol, 1977, 95: 217-23.

25. Kingham JD: Acute retrolental fibroplasia. Arch Ophthalmol 1977, 95: 39-47.

26. Keith CG: Retrolental fibroplasia, a new classification of the developing and cicatricial changes. Aust J Ophthalmol 1979, 7: 189-94.

27. Patz A: Retrolental fibroplasia (retinopathy of prematurity). Trans Ophthalmol Soc NZ 1980, 32, 49-54.

28. Biglan AW: Update on retrolental fibroplasia. Trans $P A$ Acad Ophthalmol Otolaryngol 1984, 37: 39-43.

29. Gibson DL, Sheps SB, Schechter MT, Wiggins S, McCormick AQ: Retinopathy of prematurity: a new epidemic? Pediatrics 1989, 83: 486-92.

30. Gibson DL, Sheps SB, Uh SH, Schecter MT, McCormick AQ: Retinopathy of prematurity-induced blindness: birth weight-specific survival and the new epidemic. Pediatrics 1990, 86: 405-12.

31. Nissenkorn I, Kremer I, Cohen S, Ben-Sira I: Nasal versus temporal preretinal vasoproliferation in retinopathy of prematurity. Br J Ophthalmol 1989, 73: 747-9.

32. Pollard ZF: Asymmetry in retrolental fibroplasia. Ann Ophthalmol 1983, 15: 768-71. 
33. Fielder AR, Ng YK, Levene MI: Retinopathy of prematurity: age at onset. Arch Dis Child 1986, 61: 774-8.

34. Quinn GE, Johnson L, Abbasi S: Onset of ROP as related to gestational age at birth and time from conception. BrJOphthalmol (In Press).

35. Quinn GE, Johnson L, Otis C, Schaffer DB, Bowen FW: Incidence, severity and time course of ROP in a randomised clinical trial of vitamin E prophylaxis. Doc Ophthalmol 1990, 74: 223-8.

36. Baum JD: Retinal artery tortuosity in ex-premature infants. Arch Dis Child 1971, 46: 247-52.

37. Palmer EA: Optimal timing of examination for acute retrolental fibroplasia. Ophthalmology 1981, 88: 662-8.

38. Brown DR, Biglan AW, Stretavsky M: Screening criteria for the detection of retinopathy of prematurity in patients in a neonatal intensive care unit. J Pediatr Ophthalmol Strabismus 1987, 24: 212-14.

39. Biglan AW, Cheng KP, Brown DR: Update on retinopathy of prematurity. Int Ophthalmol Clinics 1989, 29: 2-9.
40. Urrea PT and Rosenbaum AT: Retinopathy of prematurity: an ophthalmologist's perspective. In SJ Isenberg ed The eye in infancy Year Book Medical Publishers, Inc, Chicago 1989, pp. 428-456.

41. Cats BP: Retinopathy of prematurity. PhD Thesis 1990.

42. Darlow BA and Clemett RS: Retinopathy of prematurity: screening and optimal use of the ophthalmologist's time. Aust NZ J Ophthalmol 1990, 18: 41-6.

43. Fielder AR: Cryotherapy for retinopathy of prematurity. In Jay BS and Davidson SI eds. Recent advances in ophthalmology 8. Churchill Livingstone, Edinburgh 1991 pp. $129-48$.

44. Acheson JF and Schulenburg WE: Surveillance for retinopathy of prematurity in practice: experience from one neonatal intensive care unit. Eye 1991, 5: 80-5.

45. Anon: ROP screening duty. College News [Q Bull Coll Ophthalmol (Autumn)] 1990. 